\title{
ANALYSIS OF MEASURED WORKPIECE'S FORM ERRORS INFLUENCE ON THE ACCURACY OF PROBE HEADS USED ON FIVE-AXIS MEASURING SYSTEMS
}

\author{
Wiktor Harmatys' ${ }^{1}$, Adam Gąska', Maciej Gruza', Piotr Gąska', Jerzy Sładek' \\ Cracow University ofTechnology, Laboratory of Coordinate Metrology (M-10), Mechanical Engineering Faculty, \\ al. Jana Pawła II 37, 31-864 Kraków, Poland, e-mails: wiktorharmatys@gmail.com, agaska@mech.pk.edu.pl, \\ gruzam@interia.pl, piotr.gaska@pk.edu.pl, sladek@mech.pk.edu.pl
}

Received: 2017.10 .25

Accepted: 2017.11.13

Published: 2017.12.05

\begin{abstract}
The five-axis measuring systems are one of the most modern inventions in coordinate measuring technique. They are capable of performing measurements using only the rotary pairs present in their kinematic structure. This possibility is very useful because it may cause significant reduction of total measurement time and costs. However, it was noted that high values of measured workpiece's form errors may cause significant reduction of the accuracy of a five-axis measuring system. The investigation on the relation between these two parameters was conducted in this paper and possible reasons of decrease in measurement accuracy was discussed on the example of measurements of workpieces with form errors ranging from 0,5 to 1,7 millimetre.
\end{abstract}

Keywords: articulating probe head, five-axis system, accuracy, CMM

\section{INTRODUCTION}

Improvements in manufacturing techniques and production engineering along with the growing markets in automotive, aviation and machine industries cause the need for shortening the total production time of parts and components used in these branches $[10,11]$. It also creates new requirements regarding the processes that are inseparable part of production process, which include quality control or production supervision and monitoring $[7,12]$. The trends regarding decrease of measurement or inspection time are also clearly visible in these fields $[4,9]$. One of the relatively novel systems that were developed because of the abovementioned reasons are the CMM probe heads that are used in so called five-axis measuring coordinate systems. The main feature that makes them different from typical probe heads is the possibility of continuous articulation, thanks to which the five-axis measuring systems are capable of performing measurements using only probe head rotary movements [3]. This improvement gives possibility for reduction of measurement time, which is achieved because movements of heavy and large machine parts are replaced by the rapid movements of probe head rotary axes. The use of this type of probe heads helps also in reduction of CMM dynamic errors related to repeated starting and stopping of the machine movements between probing of measuring points.

Of course, apart from numerous advantages, probe heads used on five-axis measuring systems have some drawbacks $[1,5]$. They include necessity of performing long-time calibration of the head itself and all probes that are used during measurements, necessity of application of complicated error correction systems and metrological software that is compatible with that kind of probe heads. All of the above mentioned treatments are used for reducing the inaccuracies of probe heads, in order to fully use its functionalities.

Recently, it was also noted that measurement of workpieces being the solids of revolution with 
form errors reaching tenth part of millimetre may cause inaccuracies during measurements performed using five-axis systems. The experiments aiming at proving this phenomenon and the discussion on its possible reasons were performed and presented in the following sections of this paper.

\section{PREPARATION AND DESCRIPTION OF EXPERIMENTS}

The idea of conducting research presented in this paper was conceived because of authors' experiences with the use of $\mathrm{PH} 20$ probe head. It was noted that during measurements of some workpieces, the results obtained using the abovementioned probe head was visibly worse than using probe heads that function on machines working in traditional three-axis mode. The preliminary analyses done by the authors showed that this situation occurs primarily for the measured workpieces whose form errors reach tenth parts of millimetre.

In order to prove it, the experimentation was planned. It consists of four stages: modelling of material standards in shape of cylinders burdened with the predetermined values of form errors, manufacturing of them, calibration measurements performed on high accuracy CMM, measurement of manufactured standards at CMM equipped with $\mathrm{PH} 20$ probe head and comparison of calibration results with results of measurements done using $\mathrm{PH} 20$ probe.

\section{Modelling of material standards}

The cylinder-shaped material standards were modelled in chosen $3 \mathrm{~d}$ CAD software. There were two types of standards prepared. The first one (Fig. 1a) was prepared using function presented in equation (1) defined in polar coordinate system, for $\alpha$ changing in range $\left(0^{\circ}, 360^{\circ}\right)$ :

$$
y=20+0,25 * \cos 5 \alpha
$$

The curve was generated using this function and then protruded at height of $10 \mathrm{~mm}$. As can be noticed from (1), the cylinder has a diameter of $20 \mathrm{~mm}$ and the maximum negative deviation of form equals $-0,25 \mathrm{~mm}$ and maximum positive deviation of form equals $0,25 \mathrm{~mm}$, giving a total nominal form deviation equals to $0,5 \mathrm{~mm}$.

The second one (Fig. 1b) was prepared in the same way, but the base function was expressed using equation (2):

$$
y=20+0,8 * \cos 5 \alpha
$$

so the nominal form deviation of this material standard equals to $1,6 \mathrm{~mm}$.

\section{Manufacturing of material standards}

Models presented in previous section were used for printing the material standards using Fused Deposition Method (for more information on this method see [2]). Printer that was used in this process was the "Aurora $3 \mathrm{~d}$ " printer described in [6]. ABS was the material used as a filament. Figure 2 presents the process of printing the material standards and Figure 3 presents manufactured standards.

\section{Calibration of prepared standards}

Manufactured material standards were calibrated on PMM 12106 machine whose MPE errors are given by equation (3):

$$
E_{0, M P E}=0,8+2,5 * \frac{L}{1000}, \mathrm{~mm}
$$

where $L$ is the measured length given in $\mathrm{mm}$.

A calibration procedure that was used is based on methodology called "calibrated workpieces method" that was presented in [8]. As a calibrated workpiece the standard ring with diameter of 28 mm was used. 64 measuring points were used during measurements of both material standards

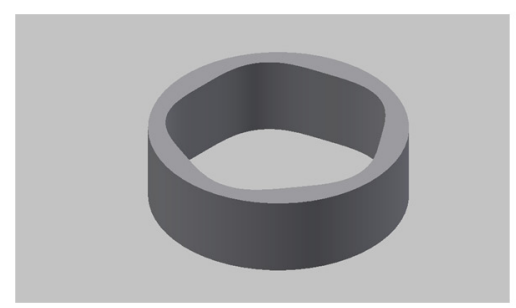

Fig. 1. CAD models of the developed material standards: a) with less significant form deformations, b) with more significant form deformations 


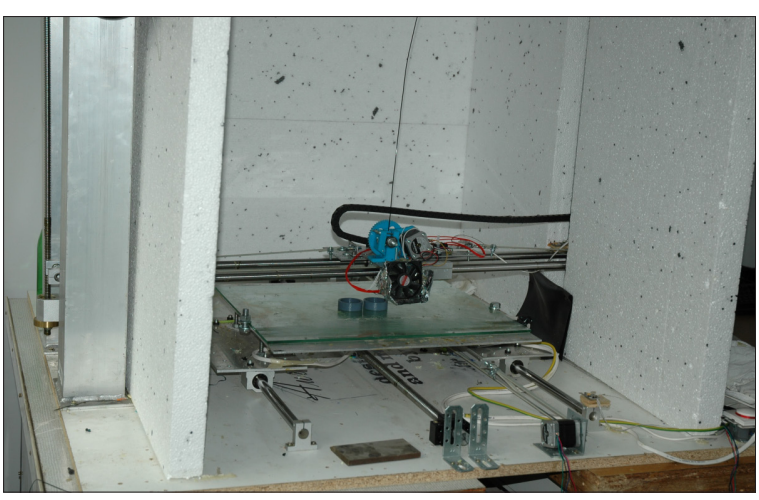

Fig. 2. Printing of material standards on "Aurora 3d" FDM printer

which were subject to calibration and calibrated workpiece. The temperature in CMM measuring volume during calibration was in the range $20 \pm$ $0,1^{\circ} \mathrm{C}$. Calibration measurements and mounting of the workpieces were presented in Figure 4.

Table 1 presents the results of calibration for both material standards.

\section{Measurements of considered workpieces}

There were 3 workpieces selected for measurements: two material standards presented in previous sections of these paper and standard ring with diameter of $20 \mathrm{~mm}$. Measurements were performed using Zeiss WMM 850s machine equipped with $\mathrm{PH} 20$ probe head. Material standards were measured in order to prove existence

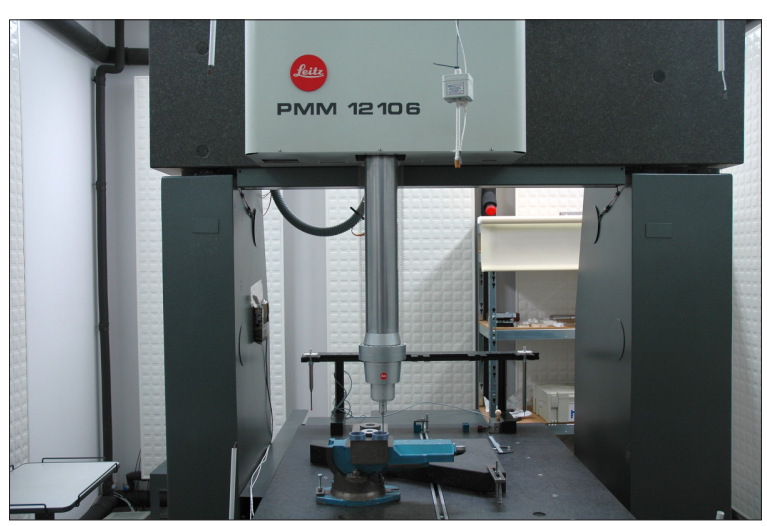

Fig. 4. Calibration measurements performed on PMM 12106 machine

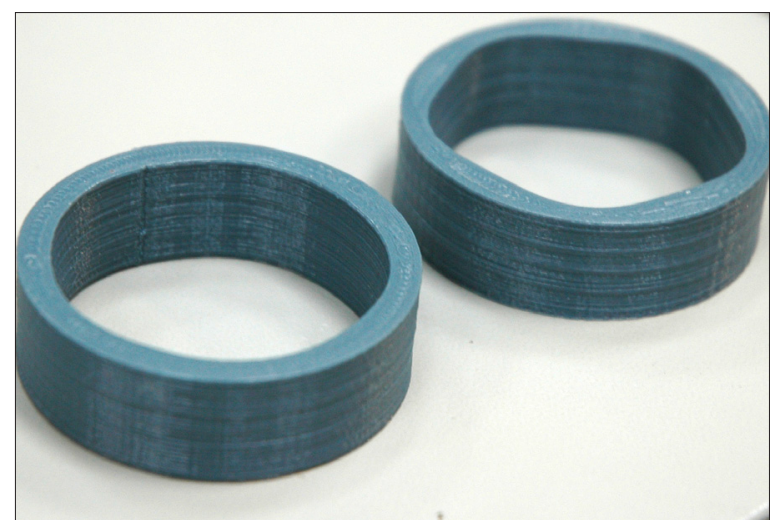

Fig. 3. Material standards prepared using $3 d$ printer

of significant accuracy loss during measurements of workpieces with high levels of form errors, while the standard ring was measured in order to prove that in a situation in which the workpieces with low values of form errors are measured the accuracy loss is not recorded. In each case, the same number and distribution of measuring points as in case of calibration measurements was used. The temperature in CMM measuring volume during measurements was in range $19,7-20,2{ }^{\circ} \mathrm{C}$.

The results obtained from measurements of all considered workpieces performed using PH20 probe head were compared with the results from calibration measurements. Measurements performed on Zeiss WMM machine are presented in Figure 5. The results of performed measurements and comparisons are shown in section 3 .

\section{RESULTS OF MEASUREMENTS}

Figures 6-8 present the results of measurements and comparisons that were described in section 2. All errors presented in these figures are mean radial errors calculated from 10 repetitions of considered measurement.

Form errors identified for standard ring by calibration measurements equaled to $0,0003 \mathrm{~mm}$ and by measurements using $\mathrm{PH} 20$ probe head to $0,0006 \mathrm{~mm}$. Deviation between these two values was $0,0003 \mathrm{~mm}$. The biggest absolute value of difference between $\mathrm{e}_{\mathrm{r}, \mathrm{PMM}}$ and $\mathrm{e}_{\mathrm{r}, \mathrm{PH} 20}$ was

Table 1. Calibration results for both manufactured material standards, eq. (1) means that the material standard was modelled using equation (1), while eq. (2) means that it was modelled using equation (2)

\begin{tabular}{|c|c|c|}
\hline Workpiece & Form deviation, $\mathrm{mm}$ & Expanded uncertainty, mm \\
\hline Material standard eq. (1) & 0,5885 & 0,0004 \\
\hline Material standard eq. (2) & 1,6806 & 0,0004 \\
\hline
\end{tabular}




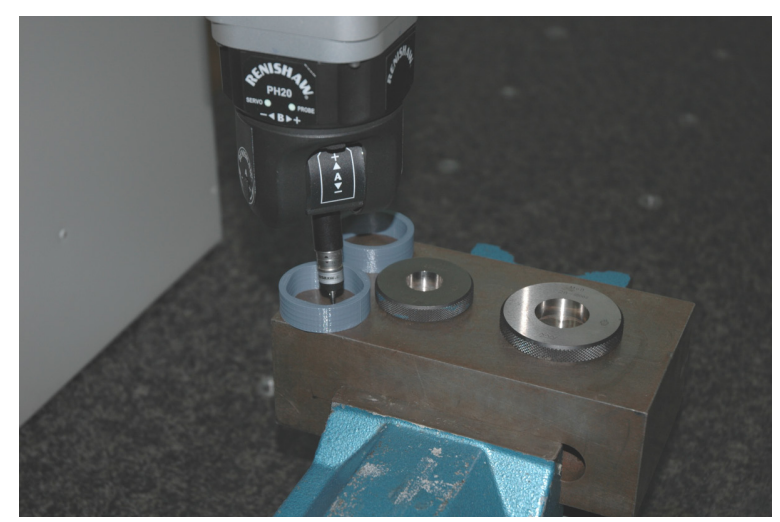

Figure 5. Measurements of material workpieces and standard ring performed on Zeiss WMM 850s machine equipped with $\mathrm{PH} 20$ probe head

achieved for $\alpha$ equal to $90^{\circ}$ and equaled 0,0004 $\mathrm{mm}$ (where $\alpha$ is the angle of approach at a certain point - it is consistent with $\alpha$ defined in equations (1) and (2), $\mathrm{e}_{\mathrm{r} \text {.PMM }}$ is the mean radial error in direction defined by $\alpha$ for calibration measurements and $\mathrm{e}_{\mathrm{r} \text {.PH } 20}$ is the mean radial error in direction defined by $\alpha$ for measurements performed using $\mathrm{PH} 20$ probe head).

Form errors identified for material standard modelled using equation (1) determined by calibration measurements equaled to $0,5885 \mathrm{~mm}$ and by measurements using $\mathrm{PH} 20$ probe head to $0,5709 \mathrm{~mm}$. Deviation between these two values was $-0,0176 \mathrm{~mm}$. The biggest absolute value of difference between $\mathrm{e}_{\mathrm{r} \text {.PMM }}$ and $\mathrm{e}_{\mathrm{r} \text {. PH20 }}$ was achieved for $\alpha$ equal to $191,25^{\circ}$ and equaled $0,0325 \mathrm{~mm}$.

Form errors identified for material standard modelled using equation (2) determined by cali- bration measurements equaled to $1,6806 \mathrm{~mm}$ and by measurements using $\mathrm{PH} 20$ probe head to $1,7060 \mathrm{~mm}$. Deviation between these two values was $0,0254 \mathrm{~mm}$. The biggest absolute value of difference between $\mathrm{e}_{\mathrm{r}, \mathrm{PMM}}$ and $\mathrm{e}_{\mathrm{r}, \mathrm{PH} 20}$ was achieved for $\alpha$ equal to $123,75^{\circ}$ and equaled $0,0519 \mathrm{~mm}$.

\section{CONCLUSIONS}

The results presented in section 3 prove the existence of accuracy loss during measurements of workpieces with high level of form error values (form errors values at the level of tenth parts of millimetre and bigger) performed using PH20 probe head. The accuracy loss is not observed for measurements of workpieces with lower values of form errors.

However, it is hard to determine unambiguous relation between the values of workpiece's form errors and values of five-axis measurement errors corresponding to them. In case of both material standards that were measured the absolute mean value of difference between the results of calibration measurements and measurements performed using PH20 probe head was equal to about 0,02 $\mathrm{mm}$. However, in case of artifact with smaller form errors, identification of their value using PH20 probe head gave smaller values than calibration, which is opposite to the measurements of artifact with bigger form errors, for which the values of form errors identified using $\mathrm{PH} 20$ probe head was bigger than in case of calibration. Having in mind the results obtained during measure-

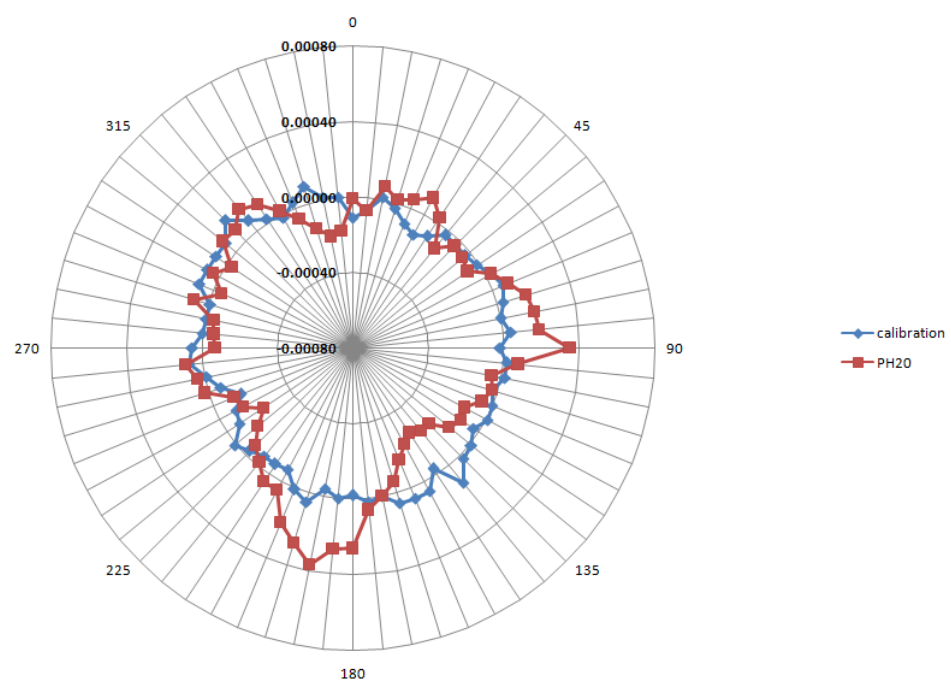

Figure 6. Graphical comparison of results of standard ring measurements. The values on vertical axis gives mean radial error in $\mathrm{mm}$, values on circumference of graph gives values of $\alpha$ angle in degrees 


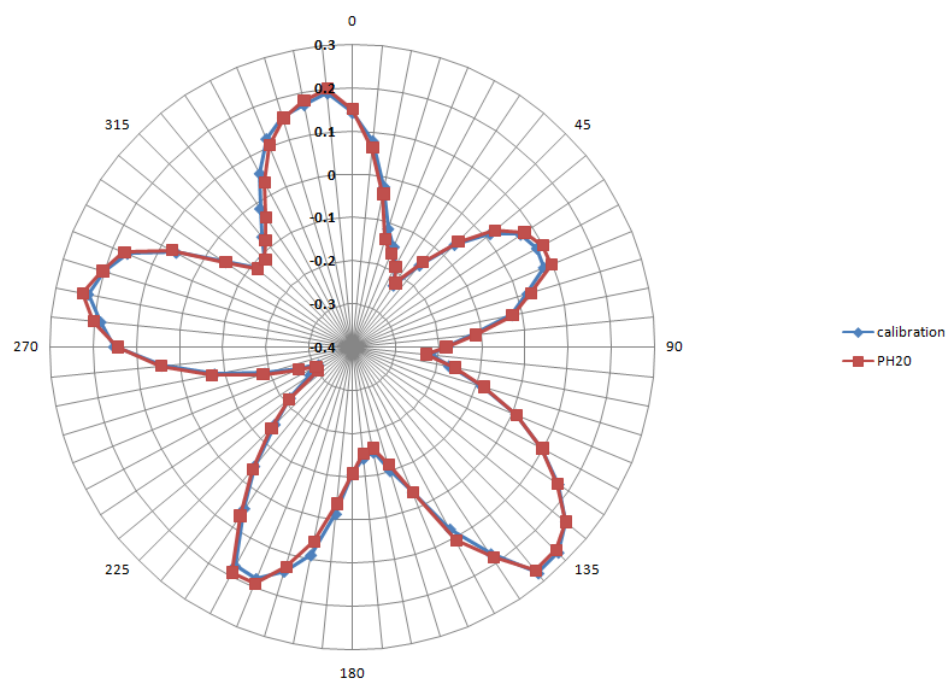

Figure 7. Graphical comparison of the results of measurements of material standard with nominal values of form error equal to $0,5 \mathrm{~mm}$. Values on vertical axis gives mean radial error in $\mathrm{mm}$, values on circumference of graph gives values of $\alpha$ angle in degrees

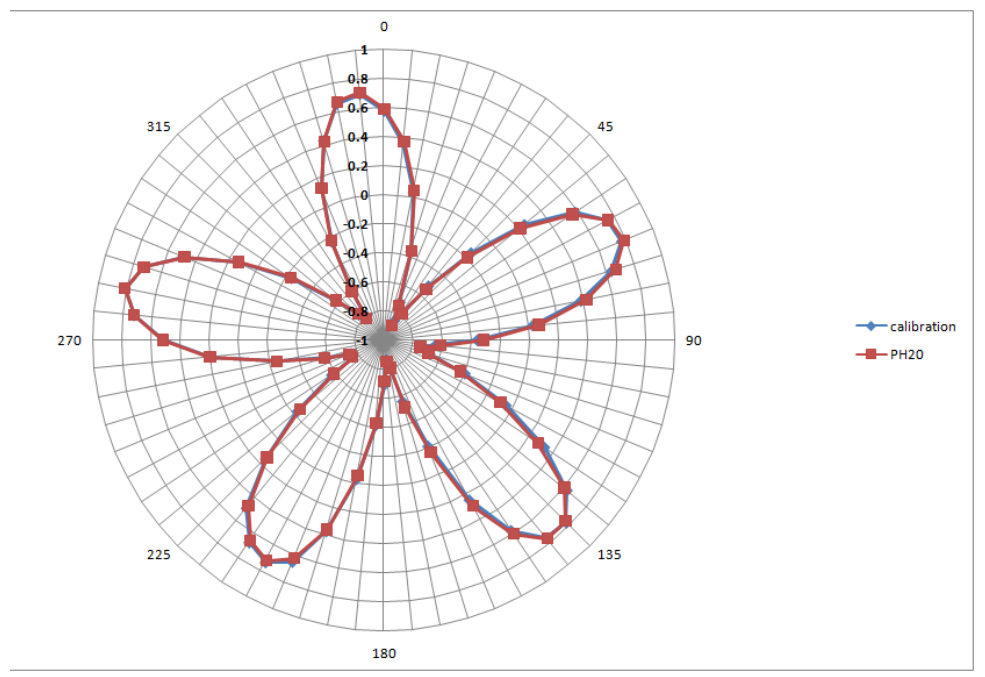

Figure 8. Graphical comparison of the results of measurements of material standard with nominal values of form error equal to $1,6 \mathrm{~mm}$. Values on vertical axis give mean radial error in $\mathrm{mm}$, values on circumference of graph give values of $\alpha$ angle in degrees

ments of standard ring, in which the difference in form errors values for both calibration and PH20 measurements was almost zero, it should be concluded that the relation between the values of workpiece's form errors and values of five-axis measurement errors corresponding to them does not have a linear character. More detailed investigations on that matter will be undertaken in near future by the authors.

As a possible cause of the observed accuracy loss, too far simplifications of algorithms used by the metrological software may be taken. Form errors in workpieces cause that the stylus mounted on probe head travels further or closer in regard to theoretical data of measured feature. In case of probe heads used on five-axis measuring systems it also means that the rotation angle in one of probe head's axes reaches higher or lower values than in case of measurement of points generated for nominal feature. It causes that the tip of stylus hits the surface of measured object in different point that is expected for generated measurement code. In this case, the radial correction of stylus tip may be done in slightly different direction that it should be and it may cause additional errors.

Identified accuracy loss is at the level of 25 micrometres for workpieces whose form errors are at the level of $1,6 \mathrm{~mm}$. So the rate of rela- 
tive accuracy loss is about $1,5 \%$. In majority of cases this value will not have significant impact on deciding whether the controlled workpiece is compliant with its geometrical specifications or not, however in some cases (when the tolerance zones are narrow), this $25 \mu \mathrm{m}$ may cause faulty decisions on that matter. So it is the CMM user's responsibility to decide if the accuracy loss should be a concern, depending on the definition of measuring task.

\section{Acknowledgments}

Reported research was realized as part of a project financed by National Science Centre, Poland, grant no. 2015/17/D/ST8/01280.

\section{REFERENCES}

1. Dobosz, M.,Woźniak, A. CMM touch trigger probes testing using a reference axis. Precision Engineering, 29, 2005, 281-289.

2. Fudali P., Witkowski W., Wydrzyński D. Comparison of geometric precision of plastic components made by subtractive and additive methods. Advances in Science and Technology Research Journal, 7(19), 2013, 36-40.

3. Gąska A., Gąska P., Gruza M. Simulation Model for Correction and Modeling of Probe Head Errors in Five-Axis Coordinate Systems. Applied Sciences, 6(5), 2016.

4. Gaska P., Gaska A., Gruza M. Challenges for modeling of five-axis coordinate measuring systems.
Applied Sciences, 7(8), 2017, 803.

5. Gruza M., Gąska A., Gąska P., Harmatys W., Sładek J. Analysis of impact of the working mode on the accuracy of the five-axis measuring system. Mechanik, 11, 2016, 1708-1709.

6. Harmatys W., Gruza M., Gąska P., Gąska A., Sładek J. The parametric model of standard for "test by work" method, adapted for 3D printers. Mechanik, 5-6, 2016.

7. Hocken R. J., Pereira P. H. Coordinate Measuring Machines and Systems, Second Edition. CRC Press, 2012.

8. ISO 15530-3:2011 - Geometrical product specifications (GPS) - Coordinate measuring machines (CMM): Technique for determining the uncertainty of measurement - Part 3: Use of calibrated workpieces or measurement standards.

9. Jóźwik J., Czwarnowski M., Angular positioning accuracy of rotary table and repeatability of fiveaxis machining centre DMU 65 MONOBLOCK. Advances in Science and Technology Research Journal, 9(28), 2015, 89-95.

10. Mayer J.R.R., Mizanur Rahman M., Los A. An uncalibrated cylindrical indigenous artefact for measuring inter-axis errors of a five-axis machine tool. CIRP Annals, 64(1), 2015, 487-490.

11. Pashaki P.V., Poulja M. Volumetric error compensation in five-axis CNC machining center through kinematics modeling of geometric error. Advances in Science and Technology Research Journal, 10(30), 2016, 207-217.

12. Weckenmann A., Estler T., Peggs G., McMurtry D. Probing Systems in Dimensional Metrology. CIRP Annals-Manufacturing Technology, 53(2), 2004, 657-684. 